\title{
Anisotropic Mechanical Properties of Materials in Stereolithographic Additive Manufacturing
}

\author{
Alan DIVJAK*, Damir MODRIĆ, Ines KOVAČIĆ, Vladimir CVILJUŠAC
}

\begin{abstract}
Rapid development and expanding deployment of additive manufacturing mark this technology as an acceptable alternative to traditional methods of production in terms of speed and quality. All types of additive manufacturing technologies build objects layer by layer, therefore anisotropy of mechanical properties is often encountered, making it difficult to accurately predict the mechanical behavior of additively manufactured parts. This paper examines the mechanical properties of additively manufactured parts and the creation of an anisotropic digital material model that can be used to accurately simulate the behavior of additively manufactured parts using FEA (Finite Element Analysis). A three-point bending test was performed on samples created using SLA (Stereolithography) technology. The identical bending scenario was computer modeled using FEA, while the modified manufacturer's data was used for digital material creation. Comparison and analysis of the experimentally obtained results and the results of the FEA were performed.
\end{abstract}

Keywords: additive manufacturing; FEA; photopolymer; stereolithography

\section{INTRODUCTION}

Additive manufacturing technology is being increasingly used in all areas of industry and is becoming a profoundly important component of the production chain. In other words, it ceases to be considered a prototyping technology and is being used for actual production. This transition has been largely facilitated by a broad range of available materials [1-3], whose diversity and quality is increasing on a daily basis. A great majority of parts produced by additive manufacturing have a functional application, meaning that they have to meet certain dimensional, mechanical and thermal requirements for the purpose that the product was intended for. Surface quality and tolerances are mostly defined by the type of additive manufacturing technology [4], while other properties are determined by the material, which further emphasizes the importance of knowing the behavior of materials in a given scenario of use in order to correctly design the part. By far the most important material characteristics are the mechanical properties that determine whether given additive manufacturing technology and materials available within this technology will be adequate to produce models that must meet a certain usage scenario. Therefore, knowledge of the real mechanical properties of the materials is essential for the successful and safe use of additive manufacturing for commercial purposes.

During the process of developing more complex additively manufactured products, the ideal situation would be to use computer simulation for part design validation, specifically using finite element analysis (FEA). Given that the entire process takes place in a computer, it is possible to quickly check the behavior of the model under certain conditions and iteratively refine the design to achieve the desired properties and design goals. In order to reach physically accurate model behavior, the key component of FEA simulations is to accurately define the loads and realistic mechanical properties of the material from which the model is made. Defining realistic mechanical properties of models is quite problematic and challenging when it comes to additive manufacturing. The problem lies in the very process of physically creating a model and it is exclusively characteristic of additive manufacturing. Additively manufactured parts are built layer by layer and in some technologies, different layers can be made in different ways. Such gradual deposition leads to the material not binding into a homogeneous mass, leading to the anisotropy of the mechanical properties [5, 6]. Materials that are anisotropic exhibit varying physical properties dependent on the direction in which a property is measured. In the case of anisotropy in additive manufacturing, there are always one or two spatial orientations (depending on load type) in which the object will have inferior mechanical properties. In addition, some technologies require extra post-processing in order to make the models fully functional and these procedures sometimes do not work uniformly on the entire model's volume. All this leads to a situation where the mechanical properties of the material declared by the manufacturer cannot be taken with complete certainty as realistic values. This paper focuses on stereolithographic additive manufacturing technology (SLA), which has many advantages over other manufacturing technologies. It is currently one of the fastest additive manufacturing technologies, achieves very high resolution, has a wide selection of materials, is easy to use, 3D printers are mechanically robust and simple, and the price of high-quality $3 \mathrm{D}$ printers is relatively low. One of the special advantages is the theoretical possibility of making objects with isotropic mechanical properties.

The aim of this paper is to determine realistic values of the material's mechanical properties as a function of spatial layer orientation using mechanical testing and to accurately recreate this material in FEA simulation for easier and faster design of additively manufactured parts. A single material was used in this research, but its behavior is indicative of the entire spectrum of materials used in the SLA additive manufacturing technology since the method of object creation is the same.

These paper's contributions help determine more realistic behavior of additively manufactured parts. Areas of application are numerous, but medicine is certainly one of the most important areas where these contributions can be applied. One of these areas is dental implantology, i.e. replacing the lost teeth with artificial tooth root inserted into the jawbone. Research presented in this paper is 
conducted within the framework of a broader research of bone and implant mechanical responses of short and slim mini dental implants (MDI) for $\mathrm{mCD}$ retention (HRZZ project, "Mini dental implants", no. 1218), using mechanical testing and Finite Element Analysis (FEA). Real-world mechanical testing of additively manufactured human mandibles (Fig. 1) with inserted commercial MDIs of various dimensions is a novel and invaluable tool in evaluating the quality and durability of dental implants.

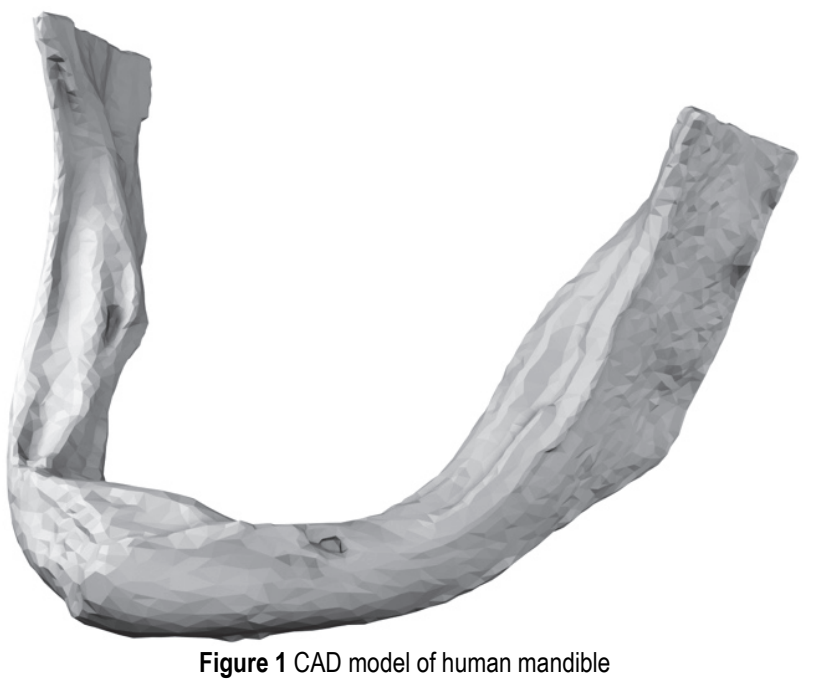

However, it is infeasible to use real human mandibles for testing, therefore additively manufactured mandibles are used. By performing mechanical testing on these facsimiles it is possible to make accurate predictions on how a real mandible would behave under load. However, to ensure the accuracy of these predictions it is necessary to know the exact mechanical properties of the material used. Therefore, mechanical characterization must be performed. These results can then be used to create FEA simulations that can often replace real-world testing in some scenarios. This dual approach is fast becoming the standard for research and development in the field of oral implantology and has numerous advantages over traditionally used methods.

\section{MATERIALS AND METHODS}

Formlabs (Somerville, Massachusetts, United States) Gray (GRAY FLGPGR04) photopolymer resin was used in this research. 3D printer Formlabs Form 2 was used for the production of 15 test specimens. Dimensions are $150 \times$ $10 \times 10$ millimeters. Groups of five test specimens were made in two different layer orientations. Two groups (a total of ten specimens) were made with the long side of the object parallel to the build plate, while one group (five test specimens) was made with the shorter side parallel to the build plate (Fig. 2). Objects were prepared for 3D print using Formlabs Preform software that comes with a Form 2 printer. and were printed with a $0.1 \mathrm{~mm}$ layer thickness.

After completing the print, models were submerged into the container with $95 \%$ isopropyl alcohol (IPA) for one minute and then transferred to a second container with 95\% IPA for 15 minutes to rinse the remaining liquid resin from printed part's surface. UV post-curing was performed in a chamber with $36 \mathrm{~W}$ UV-A halogen bulbs for 30 minutes and then placed in the heating chamber at $60{ }^{\circ} \mathrm{C}$ for 30 minutes. Such subsequent finishing is unique to SLA technology and is required to achieve maximum mechanical properties. It was done according to the manufacturer's instructions for this specific resin.

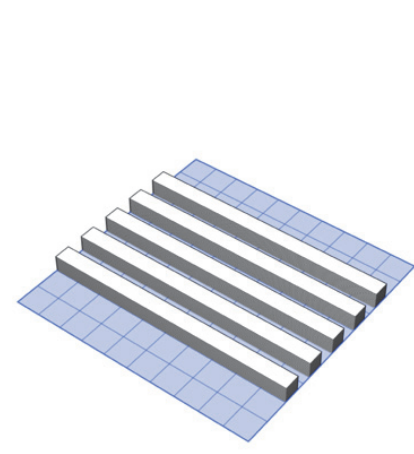

a)

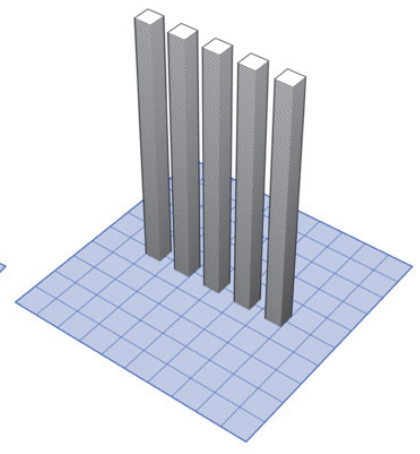

b)
Figure 2 Orientation of parts during additive manufacturing, a) group of five specimens in the horizontal orientation, b) group of five specimens in the vertical orientation

The mechanical testing was carried out in Topomatika d.o.o., where measurements were made using Hegewald \& Peschke (Nossen, Germany) INSPEKT 20-1 universal testing machine configured for a three-point bending test (Fig. 3). Fixed pins have a radius of $5 \mathrm{~mm}$, the gap between the fixed pins is $80 \mathrm{~mm}$, and the loading pin diameter is 5 $\mathrm{mm}$. Bending was performed at a rate of $5 \mathrm{~mm} / \mathrm{s}$ until the specimen failed. Force and deflection were recorded every $0.02 \mathrm{~s}$ by the universal testing machine.
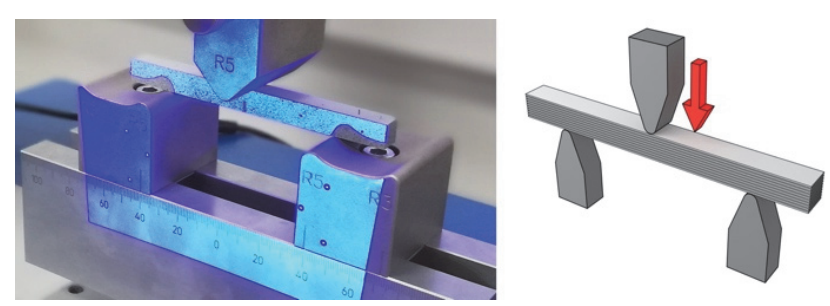

Figure 3 Three-point bending scheme

Table 1 Manufacturer's data for mechanical properties

\begin{tabular}{|l|c|c|}
\hline \multicolumn{1}{|c|}{ Tensile properties } & Green state & Post cured \\
\hline Ultimate tensile strength & $38 \mathrm{MPa}$ & $65 \mathrm{MPa}$ \\
\hline Tensile modulus & $1.6 \mathrm{GPa}$ & $2.8 \mathrm{GPa}$ \\
\hline Elongation at break & $12 \%$ & $6 \%$ \\
\hline Flexural properties & & \\
\hline Flexural modulus & $1.3 \mathrm{GPa}$ & $2,2 \mathrm{GPa}$ \\
\hline Impact properties & & \\
\hline Notched IZOD & $16 \mathrm{~J} / \mathrm{m}$ & $25 \mathrm{~J} / \mathrm{m}$ \\
\hline
\end{tabular}

The orientations of the layers of the test specimens are shown in Fig. 4.

The corresponding simulation was carried out using Ansys 16 (Canonsburg, Pennsylvania, United States) and Static Structural module. In our FE simulations, we used 8noded hexahedron elements. This type of element is commonly used in beam bending simulations, such as the one shown in this paper. Its use is appropriate given that there are no large deflections during loading that might introduce severe deformations to finite elements. The material formulation is not provided by the manufacturer since it represents the intellectual property. The finite elements were generated using the element size of $1 \mathrm{~mm}$, 
resulting in 15000 finite elements in the test specimen (Fig. 5).

a)

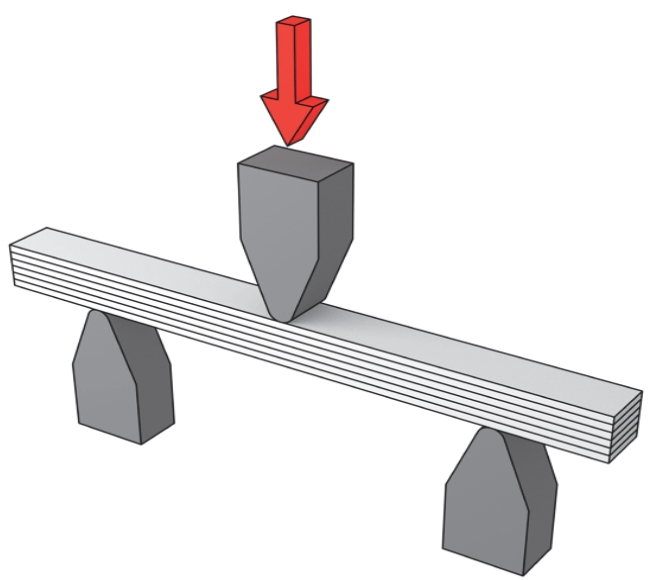

b)

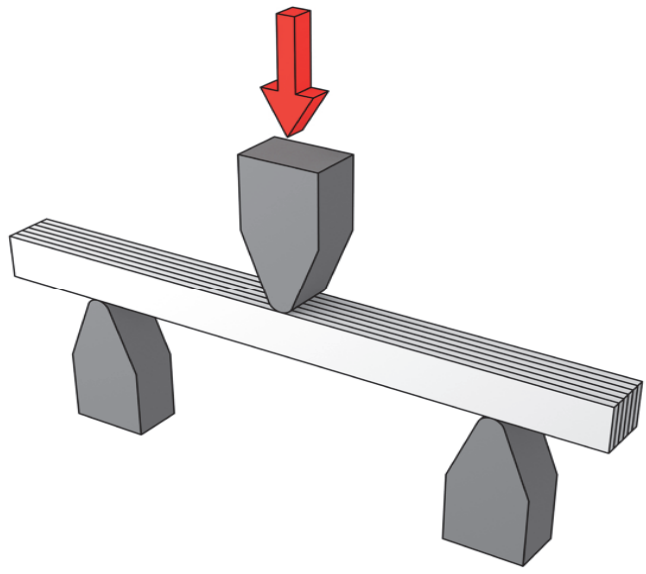

c)

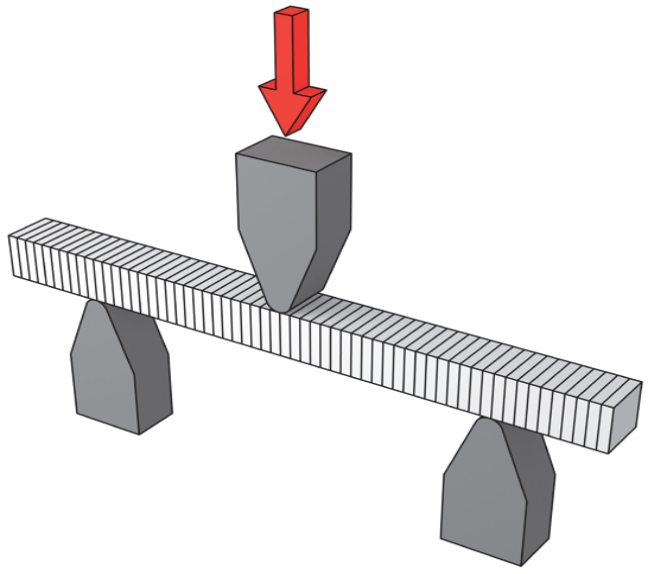

Figure 4 Orientation of the specimen and geometry of the tensile testing machine. a) horizontal layers $-\mathrm{H}, \mathrm{b}$ ) horizontal layers rotated $90^{\circ}$ about the longitudinal axis - $\mathrm{H} 90$ and $\mathrm{c}$ ) vertical layers $-\mathrm{V}$

Fixed pins were simulated using the Remote Support feature and the loading pin was simulated using the Remote Displacement feature. The samples were placed in the same position as in real-world testing, while the manufacturer's data for the mechanical properties were used (Tab. 1). The bending rate was $5 \mathrm{~mm} / \mathrm{s}$. Solver settings were slightly modified for faster solution convergence. The iterative method was used and the solver was configured with 5 minimum steps, 20 initial steps, and 50 maximum steps. The maximum load used is $300 \mathrm{~N}$ since this is the established nominal force used in mastication [7], therefore this range of forces is of interest.

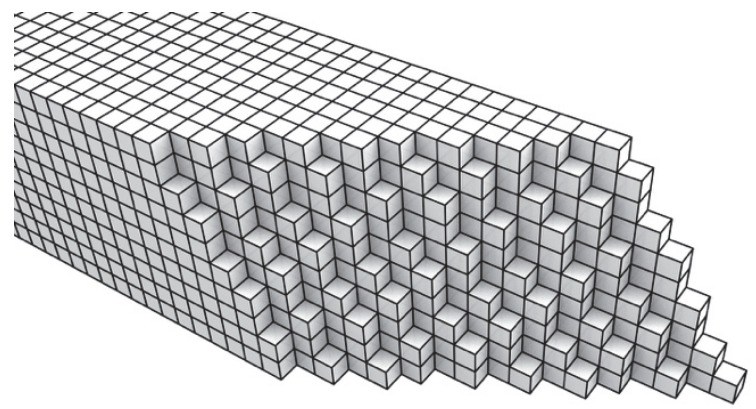

Figure 5 Cross-section of a specimen with finite elements shown

\section{RESULTS AND DISCUSSION}

Stereolithography selectively spatially polymerizes liquid photopolymer resin with an appropriate light source, typically a laser or UV light-emitting diode having sufficient radiation power in the range of $360-420 \mathrm{~nm}$, although the wavelength of $405 \mathrm{~nm}$ is most commonly used. The original method that is still widely used today is the use of a laser beam that plots the cross-section of each layer in a thin layer of liquid photopolymer. Newer methods use masks made by LCD screens or DLP DMD (Digital Light Processing Digital Micromirror Device) chips to simultaneously illuminate the entire cross-section of the object. Stereolithography is essentially a chemical process, so in theory, the chemical bonds between the layers should be as strong as those within a single layer [8]. In other words, the object should be isotropic in terms of mechanical properties. The problem arises because the material inside the object is not completely polymerized during additive manufacturing, but needs to be subsequently placed in the UV curing chamber to achieve the maximum mechanical properties.

Resins can consist of chains with lengths ranging from one to several thousand carbon atoms; the longer the chain length, the more viscous the resin. Photoreactive resins designed for additive manufacturing contain three key ingredients that enable the conversion of liquid resin to solid plastic parts while achieving high-resolution prints. These ingredients are monomers and oligomers, photoinitiators, and additives. SLA printers use a laser to selectively cure a 3D object one point at a time by drawing its cross-section through a transparent, PDMS (silicone) window [9]. When an object is formed using SLA technology, liquid resin composed of monomers or oligomers forms a cross-linked network of polymer chains that are held together with covalent bonds. During the printing process, each layer is cured using the minimum dosage of UV radiation required to convert liquid resin into solid. The 3D part that emerges is in a semi-cured "green state" that is much softer and more flexible than the fully cured part. Because layers are produced in a semi-cured green state and polymerization reactions are not driven to completion, each layer has remaining polymerizable groups on its surface that can then bond to the subsequent layer. As the next layer is formed, cross-linked polymer chains are formed between the current and previous layers forming a polymer network. Subsequent post-curing finishes polymerization process and on the molecular level polymer network in principle shows no difference between $X, Y$, and $Z$ directions and is isotropic. 

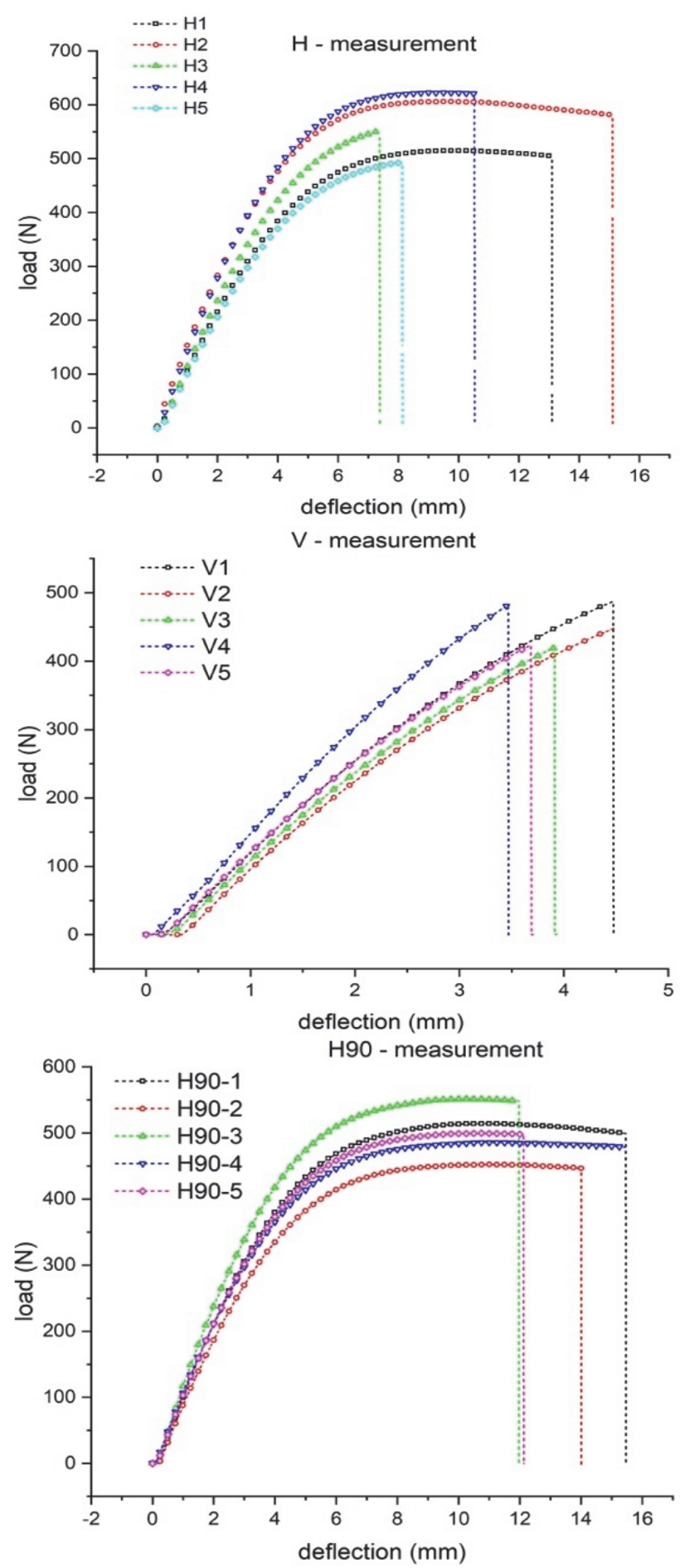

Figure 6 Load-deformation diagrams for three different orientations of the specimens (see Fig. 4)

The above-described materials characterization experiment gave the following load-deformation data with the results shown in Fig. 6. Load-deformation data obtained from tensile and/or compressive tests do not give a direct indication of the material behavior, because they depend on the specimen geometry. However, since the geometry of all three samples was the same, the only difference was the part orientation during printing and testing. Manufacturer's mechanical properties data was used for initial simulation and modification to material's mechanical properties was applied since slight anisotropy was encountered. In order to match experimentally obtained data, elastic modulus for each material orientation had to be modified in order to correspond to measured data (Tab. 2), resulting in a material model that accurately describes material's real-world behavior. Values presented in diagrams are mean values of five mechanical testings for each layer orientation.

Table 2 Manufacturer's and modified elastic modulus

\begin{tabular}{|c|c|c|}
\hline \multicolumn{3}{|c|}{ Elastic modulus } \\
\hline Specimen orientation & Manufacturer's value & Modified value \\
\hline H & $2.8 \mathrm{GPa}$ & $1.7 \mathrm{GPa}$ \\
\hline $\mathrm{H} 90$ & $2.8 \mathrm{GPa}$ & $1.45 \mathrm{GPa}$ \\
\hline $\mathrm{V}$ & $2.8 \mathrm{GPa}$ & $1.8 \mathrm{GPa}$ \\
\hline
\end{tabular}

A typical load-deformation curve is shown in Fig. 6. The load and deformation were proportional in the initial portion of the curve. The longitudinal elastic modulus of each specimen was calculated from the slope of the linear elastic portion of the curve [10]:

$$
E=\frac{1}{2} \cdot \frac{F}{d} \cdot \frac{a\left(3 l^{2}-4 a^{2}\right)}{b h^{3}}
$$

where: $F$ - load, $d$ - deformation, $l$ - distance between supports, $a$ - distance from support to point of load, $b$ width of the specimen, $h$ - thickness of the specimen.

From Eq. (1), it is apparent that the strain is not directly measured as is the case in an axial tension or compression test. The deformations in a bending test, however, are much larger and are therefore more easily measured than in the axial test. The results are shown in Tab. 3 .

Table 3 Comparative table of elasticity coefficients $(\mathrm{N} / \mathrm{mm})$ for all three-layer orientations

\begin{tabular}{|c|c|c|}
\hline \multicolumn{3}{|c|}{ Elasticity coefficient / N/mm } \\
\hline Layer orientation & Experiment / mean & FEA \\
\hline H & 120.26 & 117.37 \\
\hline H90 & 103.68 & 99.71 \\
\hline V & 124.55 & 134.65 \\
\hline
\end{tabular}

The research was limited to modeling material behavior under conditions that are encountered in a realistic situations. This implies that the loads will be relatively small and will remain in the elasticity range. Namely, we are not able to carry out realistic research of human mandibular behavior with embedded implants under real loads and conditions [11]. Therefore, we test and model these behaviors on additively manufactured models. However, these models are produced from the specific plastic so we must make a detailed characterization of the material used to properly incorporate well-known bone parameters into our models and thus generate models and behaviors of realistic mandibles using FEA model-based approaches. A major indicator of the functional state of the masticatory system is the generated biting force [12]. Edentulous patients with conventional removable dentures do not generate high biting forces as the dentate patients, or as patients with implant-supported overdentures [13]. Also, there is a difference between genders in biting forces, the mean maximum bite force for man was $847 \mathrm{~N}$ and for women $597 \mathrm{~N}$ (recordings were taken in the molar region). Therefore, we want to ascertain whether knowledge of the behavior of the used material can give us useful knowledge and experience in testing realistic mandibles printed with SLA additive technology and their interactions with 
inserted commercial implants for mandibular overdenture retention, especially because the focus is on the geriatric patients with the extremely atrophied mandible. Namely, 3D modeling of the material is not enough for a good result, but we have to pay attention to the preparation of the printing (layer orientation) and post-curing.

The results presented in Fig. 7 show that the FEA analysis provides excellent results in the area of interest (correlation $\sim 99 \%$ ).

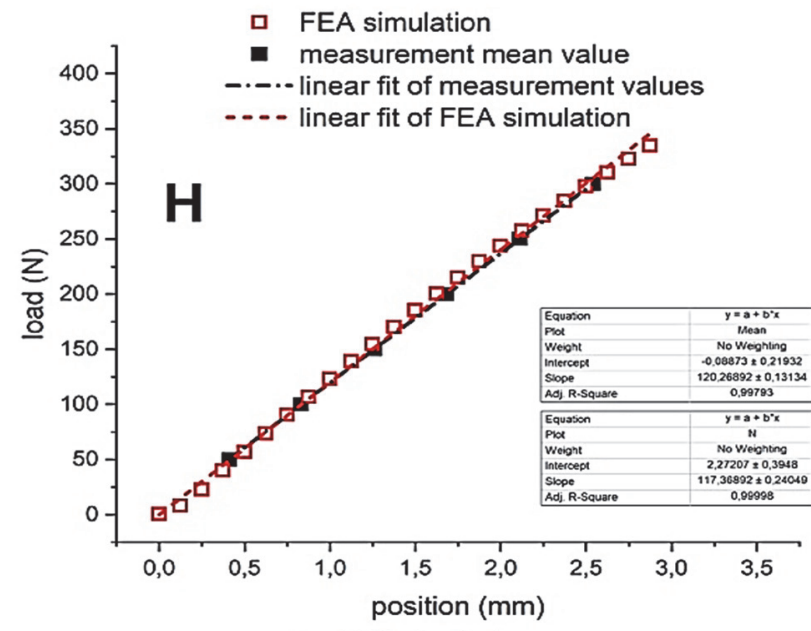

- FEA simulation

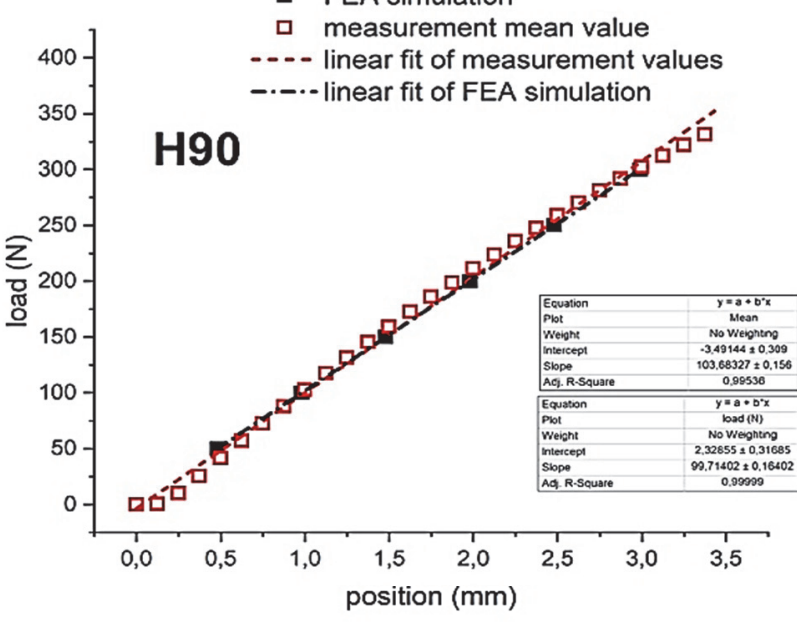

FEA simulation

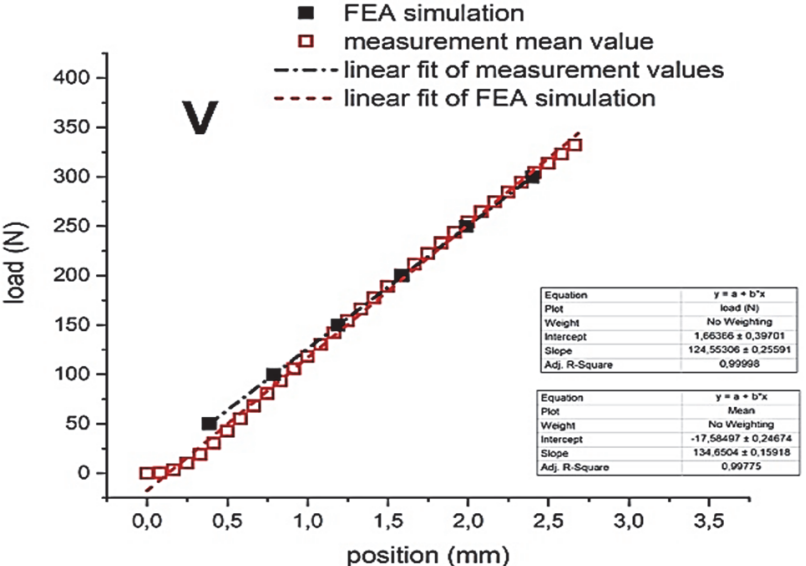

Figure 7 Hooke's law is $F=-k x$ where $F$ is the applied force $(\operatorname{load}(N)), x$ is the deformation, and $k$ a constant for a particular specimen

Fig. 8 shows that there are no significant differences in the behavior of our specimens under load depending on the orientation of the layers. However, it is necessary to emphasize that in our realistic mandibular models MDI's will be inserted later. Such action can lead to the local overstressing of the material, which ultimately can lead to the destruction of the model itself.

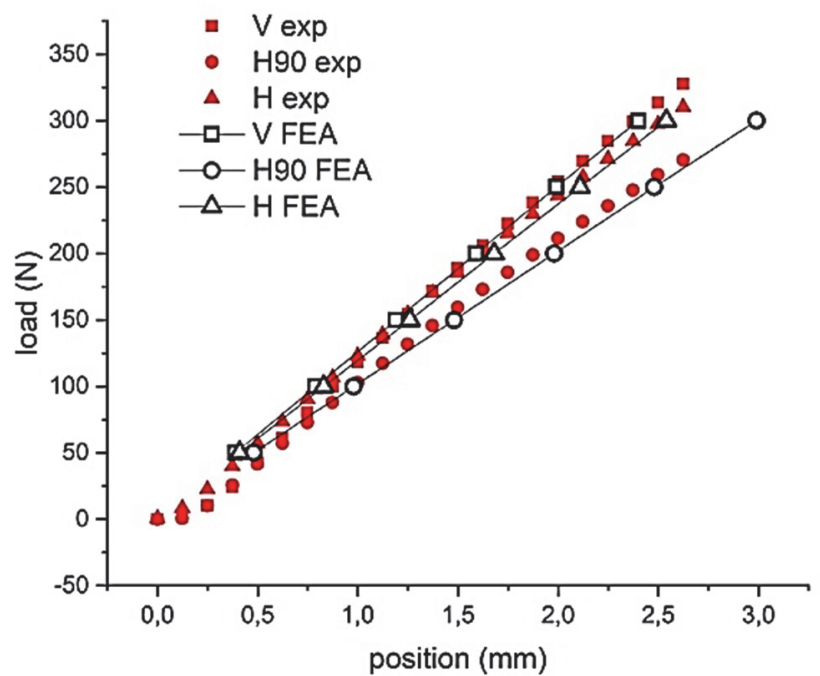

Figure 8 Comparison of experimental results and associated FEA models for all three-layer orientations

\section{CONCLUSION}

Additive manufacturing is the process of creating a physical object based on its virtual representation by adding material layer by layer. The parts produced are dimensionally accurate and can be robust in their mechanical properties; however, the build orientation and the used material can significantly affect elastic modulus and fracture stress [14]. These tests showed that, in the load range of interest, the part's production orientation has a relatively insensitive effect on elastic modulus and fracture stress.

Bite force may be an important parameter in implant selection and in the planning of prosthetic restoration, especially in patients who are capable of delivering very high occlusal loads [15]. A high bite force can result in bone resorption around implants or most commonly, fractured implant components or abutment unscrewing.

The orientation of the layers does not show a significant difference in the area of interest (Fig. 8), but we still consider preferable the orientation of the layers marked with letter $\mathrm{H}$, since MDI may be able to split layers during its insertion and thus weaken the entire structure. The first FEA results indicate promising results, but our research is continuing in the direction of the realization of the usable additively manufactured human mandible to study the mechanical consequences of MDI's embedding. Under the proposed model it is assumed that it will be possible to determine the stresses in the real mandible. Ultimately, it is intended that such testing could be performed for an individual patient in order to optimally incorporate MDI.

\section{Acknowledgments}

The authors thank the Croatian Science Foundation for funding the project ("Mini Dental Implants" No.1218). The authors report no conflicts of interest. 


\section{REFERENCES}

[1] Travitzky, N., Bonet, A., Dermeik, B., Fey, T., FilbertDemut, I., Schlier, L., Schlordt, T., \& Greil, P. (2014). Additive manufacturing of ceramic-based materials. Advanced Engineering Materials, 16(6), 729-754. https://doi.org/10.1002/adem.201400097

[2] Gu, D. D., Meiners, W., Wissenbach, K., \& Poprawe, R. (2012). Laser additive manufacturing of metallic components: materials, processes and mechanisms. International materials reviews, 57(3), 133-164. https://doi.org/10.1179/1743280411Y.0000000014

[3] Bourell, D., Kruth, J. P., Leu, M., Levy, G., Rosen, D., Beese, A. M., \& Clare, A. (2017). Materials for additive manufacturing. CIRP Annals, 66(2), 659-681. https://doi.org/10.1016/j.cirp.2017.05.009

[4] Turner, B. N. \& Gold, S. A. (2015). A review of melt extrusion additive manufacturing processes: II. Materials, dimensional accuracy, and surface roughness. Rapid Prototyping Journal, 21(3), 250-261. https://doi.org/10.1108/RPJ-02-2013-0017

[5] Carroll, B. E., Palmer, T. A., \& Beese, A. M. (2015), Anisotropic tensile behavior of Ti-6Al-4V components fabricated with directed energy deposition additive manufacturing. ActaMaterialia, 87, 309-320. https://doi.org/10.1016/j.actamat.2014.12.054

[6] Lee, C. S., Kim, S. G., Kim, H. J., \& Ahn, S. H. (2007) Measurement of anisotropic compressive strength of rapid prototyping parts. Journal of materials processing technology, 187, 627-630. https://doi.org/10.1016/j.jmatprotec.2006.11.095

[7] Nickel, J. C., Iwasaki, L. R., Walker, R. D., McLachlan, K. R., \& McCall Jr, W. D. (2003). Human masticatory muscle forces during static biting. Journal of dental research, 82(3), 212-217. https://doi.org/10.1177/154405910308200312

[8] Cheah, C. M, et al. (1997). Characteristics of photopolymeric material used in rapid prototypes Part II, Mechanical properties at post-cured state. J Mater Process Technol, 67(13), 46-49. https://doi.org/10.1016/S0924-0136(96)02816-6

[9] J. R. T. A. L. Q. S. J. M. J. M. D. Rima Janusziewicz (2016). Layerless fabrication with continuous liquid interface production. Proceedings of the National Academy of Sciences of the United States of America, 113(42), 1170311708. https://doi.org/10.1073/pnas.1605271113

[10] Timoshenko, S. P. \& Gere, J. M. (1963). Theory of Elastic Stability. McGraw-Hill, International Student Edition, Second Edition, ISBN 0-07-Y85821-7.

[11] Fernandes, C. P., Glantz, P. O., Svensson, S. A., \& Bergmark, A. (2003). A novel sensor for bite force determinations. Dent Mater, 19(2), 118-126. https://doi.org/10.1016/S0109-5641(02)00020-9

[12] Kleinfelder, J. W. \& Ludwigt, K. (2002). Maximal bite force in patients with reduced periodontal tissue support with and without splinting. J Periodontol, 73(10), 1184-1187. https://doi.org/10.1902/jop.2002.73.10.1184

[13] ASTM F2792-12a. (2012). Standard terminology for additive manufacturing technologies. West Conshohocken, PA: American Society for Testing and Materials International.

[14] Cazon, A., Morer, P., \& Matey, L. (2014). PolyJet technology for productprototyping: tensile strength and surface roughness properties. P I MechEng B J Eng, 228, 1664-75. https://doi.org/10.1177/0954405413518515

[15] Flanagan, D. (2017). Bite force and dental implant treatment: a short review. Med Devices (Auckl), 10, 141-148. https://doi.org/10.2147/MDER.S130314

\section{Contact information:}

Alan DIVJAK, mag. ing. techn. graph (Corresponding author)

University of Zagreb, Faculty of Graphics Arts Getaldićeva 2, 10000 Zagreb, Croatia

E-mail: alan.divjak@grf.hr

Damir MODRIĆ, izv.prof.dr.sc

University of Zagreb, Faculty of Graphics Arts, Getaldićeva 2, 10000 Zagreb, Croatia

E-mail: damir.modric@grf.hr

Ines KOVAČIĆ, D.M.D

Department of Removable Prosthodontics,

School of Dental Medicine, University of Zagreb,

Gundulićeva 5, 10000 Zagreb, Croatia

E-mail: ikovacic@sfzg.hr

Vladimir CVILJUŠAC, mag. ing. techn. graph University of Zagreb, Faculty of Graphics Arts, Getaldićeva 2, 10000 Zagreb, Croatia

E-mail: vladimir.cviljusac@grf.hr 